\title{
FORME DELLA PSICOANALISI E TEORIA DEL RICONOSCIMENTO. LA PSICHE INTERSOGGETTIVA DI AXEL HONNETH*
}

\author{
Marco Solinas $^{* *}$
}

È stato Axel Honneth, allievo del celebre Jürgen Habermas ed erede della sua cattedra francofortese di filosofia sociale, ad aver rilanciato a più riprese quella alleanza teoretica tra la "teoria critica" e la psicoanalisi che aveva caratterizzato il programma originario della cosiddetta Scuola di Francoforte. Indirizzo di ricerca reso celebre dai contributi di figure del calibro di Max Horkheimer, Theodor Adorno, Herbert Marcuse, Erich Fromm e Walter Benjamin, che avevano ruotato attorno all'Istituto per la Ricerca Sociale di Francoforte, istituto di cui Honneth è stato poi a lungo direttore. Al fine di rinnovare la sinergia tradizionale, Honneth - considerato uno dei più autorevoli esponenti della cosiddetta terza generazione francofortese - ha riallacciato la sua teoria del riconoscimento al pensiero psicoanalitico in forme, modalità e sensi molteplici. Egli ha ad esempio tentato di farsi carico della sfida postmoderna lanciata alla metapsicologia tradizionale dal punto di vista della pluralizzazione e fluidificazione delle strutture identitarie coadiuvata dalla dissoluzione di ruoli, relazioni e figure sociali tradizionali. Così come si assiste al tentativo reiterato di riattualizzare sul doppio piano psicopolitico e morale la nozione di angoscia nevrotica, svincolandola dalla rigidità dell'originario dualismo pulsionale freudiano e presentandola quale fenomeno ancorato alla separazione da una totalità originaria. Più in generale, Honneth negli ultimi anni ha intrapreso un cammino volto a una rilettura degli schemi psicoanalitici inerenti ai conflitti intrapsichici in chiave di riconoscimento e dialogo: ha cercato

\footnotetext{
* Il presente articolo è una rielaborazione della relazione tenuta ai "Seminari Internazionali di Psicoterapia e Scienze Umane” di Bologna il 21 febbraio 2015. Ringrazio i partecipanti per la stimolante discussione e in particolare Pier Francesco Galli per i preziosi commenti storicoanalitici.

** Via dei Pecori 1, 50123 Firenze, E-Mail <mrc.solinas@gmail.com>.

Psicoterapia e Scienze Umane, 2015, XLIX, 2: 221-242. $\quad$ DOI: 10.3280/PU2015-002004 http://www.psicoterapiaescienzeumane.it

ISSN 0394-2864
} 
di ricollocare le dinamiche pulsionali entro un quadro psichico spaziale complessivo ridisegnato dalla prospettiva delle strutture delle forme di comunicazione e della intersoggettività. Ma è significativa anche l'operazione originaria volta a reinterpretare le forme hegeliane del reciproco riconoscimento inerenti alla sfera dell'amore e degli affetti primari ricorrendo ai modelli interpersonali adottati nell'ambito della teoria delle relazioni oggettuali, con particolare riferimento ai processi della formazione identitaria.

Queste sono del resto soltanto alcune delle linee di ricerca che, in un arco temporale ormai più che ventennale, Honneth si è impegnato a portare avanti in relazione alla tradizione psicoanalitica. Egli ha pertanto dischiuso sia degli inediti percorsi a essa interni, volti a innovarla innestando in essa nuovi elementi ripresi dal proprio framework intersoggettivista, sia delle traiettorie che muovono in direzione inversa, volte ad acquisire il portato di alcuni particolari indirizzi psicoanalitici nel quadro della propria teoria del riconoscimento. Si tratta pertanto di un autore il cui approccio al pensiero psicoanalitico è poliforme e polivalente, e certamente segnato dalla volontà di affinare categorie e griglie concettuali in relazione alle trasformazioni storiche delle società contemporanee adoperando una serie di indirizzi teoretici avanguardistici della filosofia sociale, morale e politica. Per affrontare con sistematicità e linearità l'eterogeneità degli approcci e la ricchezza dei temi che Honneth ha sviluppato o a volte anche soltanto abbozzato nei confronti del pensiero psicoanalitico nel corso del tempo, possiamo scandire tale percorso in tre fasi principali, connotate dall'adozione di particolari posture metodologiche.

In un primo momento, che possiamo condensare nella stesura di Lotta per il riconoscimento (del 1992), la fondazione di taglio intersoggettivistico di un framework filosofico-sociale incentrato sul concetto hegeliano del reciproco riconoscimento ha trovato nella psicoanalisi un sostegno significativo, seppur non essenziale. Ripresa la partizione hegeliana del riconoscimento nei tre stadi dell'amore, del diritto e della solidarietà, Honneth ha articolato il primo stadio utilizzando in modo deciso la strumentazione concettuale costruita da Winnicott, e poi gradualmente sviluppata dai successivi teorici delle relazioni oggettuali. Teoria che è così venuta ad affiancarsi al modello intersoggettivistico del processo della costruzione identitaria ancorato alla psicologia sociale di George Herbert Mead. La prima ha coperto per così dire il lato lasciato scoperto da quest'ultima: quello inerente alla dimensione affettiva delle relazioni primarie. Tale ricorso alla tradizione inaugurata da Winnicott si è nel contempo accompagnato a una critica piuttosto dura, seppur relativamente marginale, di Freud e dell'indirizzo più ortodosso dei suoi interpreti, filone accusato per l'appunto di trascurare la dimensione intersoggettiva della costruzione del Sé infantile, e di privilegiare al contrario l'immagine di un bambino narcisisticamente chiuso in se stesso. 
In un secondo momento, i cui inizi si possono indicativamente ricondurre al saggio del 1999 "Teoria delle relazioni oggettuali e identità postmoderna”, Honneth ha come rovesciato la precedente prospettiva metodologica. Egli ha iniziato a lavorare sulla psicoanalisi non solo cercando in essa indirizzi e movimenti che potessero offrire un ulteriore sostegno al proprio framework teoretico, ma mirando sincronicamente a traslare e a tradurre le sue griglie interpretative all'interno delle teorie psicoanalitiche; ha incominciato per così dire a esportare la propria strumentazione concettuale. Alcune delle dinamiche basilari del funzionamento di una psiche intesa in chiave psicoanalitica sono state così rilette quali forme peculiari di relazioni di comunicazione e di riconoscimento. Honneth ha pertanto deliberatamente proiettato le forme e le strutture delle dinamiche relazionali esterne, lette attraverso le griglie dell'interazionismo proprie della teoria sociale del riconoscimento, sulle architetture e i meccanismi inerenti agli eventi e al funzionamento della psiche. La psiche individuale è stata così intesa nei termini delle strutture delle relazioni comunicative e di reciproco riconoscimento adottate per interpretare la realtà sociale.

È stato sempre insistendo sul modello intersoggettivistico proprio della teoria delle relazioni oggettuali che Honneth ha poi insistito con decisione nel ricondurre anche la sfera negativa dell'aggressività, delle energie anti-sociali, tradizionalmente ricondotta alle pulsioni di morte, non a un ipotetico sostrato bio-antropologico, ma appunto ai primi processi relazionali del bambino e alle forme di socializzazione. È in questo contesto che si colloca il serrato dibattito avviato agli inizi del XXI secolo con lo psicoanalista statunitense Joel Whitebook, per molti aspetti ben più vicino all'ortodossia freudiana. Un dibattito durante il quale Honneth ha proseguito nella sua opera di costruzione sistematica di un framework in cui la "teoria critica" (l'impostazione teoretica di filosofia sociale varata originariamente dalla cosiddetta Scuola di Francoforte) e la psicoanalisi si ritrovano a lavorare congiuntamente su un doppio fronte: rinforzandosi e sorreggendosi vicendevolmente. Ed è in tal senso emblematico che un articolo del 2001 intitolato "Il lavoro del negativo" nella prima edizione avesse come sottotitolo "Una revisione psicoanalitica della teoria del riconoscimento", mentre nella successiva edizione Suhrkamp esso reciti "Una revisione in chiave di teoria del riconoscimento della psicoanalisi”. La relazione tra i due poli teoretici è evidentemente circolare.

Posti i primi elementi volti a sviluppare un discorso di reciproca validazione dei due impianti teoretici in gioco, di lì a poco (a partire dal 2002) Honneth ha mostrato di voler sviluppare una terza prospettiva metodologica in relazione alla tradizione psicoanalitica: quella della psicologia politica - posto peraltro che nel contempo egli si è diretto con sempre maggior decisione verso l'ambito della filosofia politica. Determinante in tal senso la ripresa della nozione freudiana di angoscia, fondamentale già per la prima generazione della 
teoria critica francofortese. Ricollocata nell'alveo della teoria delle relazioni oggettuali, l'angoscia è stata difatti adoperata come chiave interpretativa di una serie di patologie sociali dalle chiare valenze e ascendenze politiche. La psicoanalisi è stata così reinnestata in uno degli assi portanti di una teoria critica della società rinnovata: sia sul versante diagnostico-analitico dei fenomeni regressivi di massa, sia su quello terapeutico-normativo. Honneth ha pertanto rilanciato il progetto originario, seppur rivisto nei contenuti e nelle forme specifiche, di alleanza teoretica e di collaborazione sinergica tra teoria critica e psicoanalisi.

È sempre su questo doppio piano e nella medesima direzione che Honneth ha continuato a muoversi in due articoli datati 2006: l'uso psicopolitico di talune forme della psicoanalisi è stato ulteriormente affinato. È stato difatti chiarito come determinati strumenti offerti dalla psicoanalisi possano contribuire a individuare quegli atteggiamenti interiori, quelle forme peculiari di relazione con se stessi che inibiscono più o meno indirettamente la stabilità e la vitalità di uno stato democratico di diritto. È stato pertanto rilanciato anche da questa prospettiva il tentativo di tradurre le dinamiche intrapsichiche nei termini di una forma di riconoscimento di particolari desideri e pulsioni tale da permettere lo sviluppo di un modello dialogico intrapsichico improntato alla tolleranza, il cui fine ultimo sarebbe l'ampliamento della sfera della libertà interiore. È anche in virtù di questo approccio che Honneth ha potuto riaprire un canale di confronto, seppur sempre critico, con il progetto emancipatorio di ampliamento della sfera della volontà riscoperto direttamente in Freud. Una sorta di parziale e se vogliamo tardiva riconciliazione con il padre fondatore della psicoanalisi ribadita anche in interventi più recenti.

È seguendo questa scansione cronologica che ho articolato il presente contributo, volto essenzialmente a offrire una panoramica complessiva delle principali modalità di lettura, dei differenti usi e delle riletture della psicoanalisi proposte da Honneth in relazione alla sua teoria del riconoscimento nel corso del tempo. Schematicamente, la prima parte è così dedicata alla delineazione della prospettiva in virtù della quale la teoria delle relazioni oggettuali viene $\mathrm{a}$ configurarsi come una delle forme peculiari del reciproco riconoscimento. Framework di cui vengono preliminarmente forniti i primi gradi delle coordinate concettuali della teoria hegeliana di fondo. La seconda parte concerne un'analisi delle direttrici seguite nel tentativo di traslare in taluni aspetti del quadro intrapsichico di matrice psicoanalitica il modello teoretico comunicativo e intersoggettivo proprio della teoria del riconoscimento, e di alcune conseguenze di tale operazione in relazione alla tematizzazione delle pulsioni. Ma si tiene conto anche dell'uso della psicoanalisi mirato a ridelineare alcuni aspetti salienti della strumentazione concettuale e delle griglie teoretiche di una teoria critica della società rinnovata. La terza parte verte infine sugli sviluppi di tale ultimo orientamento, con particolare riferimento alla diagnosi delle pa- 
tologie sociali, e all'ausilio della psicoanalisi per conferire alla teoria critica dei tratti normativi, sempre sul doppio piano politico e sociale. Operazione questa che ha comportato un graduale riavvicinamento ad alcuni dei tratti salienti dell’impostazione originaria dello stesso Freud.

\section{Le relazioni oggettuali all'interno della teoria intersoggettiva del reci- proco riconoscimento}

\section{L'approccio intersoggettivista all'identità individuale}

È attraverso il reciproco riconoscimento che nasce, si sviluppa e si consolida l'identità individuale. È questo il cuore intersoggettivista della concezione del giovane Hegel, soprattutto degli scritti del periodo di Jena (1801-1807), ripresa da Honneth (1992) in Lotta per il riconoscimento. Al centro dell'analisi sono anzitutto le forme specifiche e i dinamismi peculiari della relazione che viene a costituirsi tra due (o più) soggetti, e non semplicemente le caratteristiche tra due soggetti interrelati. Il concetto stesso di riconoscimento rimanda del resto direttamente e costitutivamente a una logica relazionale interpersonale. L'elemento della reciprocità radicalizza nel contempo tale carattere: i due poli della relazione sono come racchiusi in un gioco di specchi. Il soggetto percepisce infatti alcuni tratti (facoltà o qualità) di se stesso, e quindi procede nel processo di costruzione identitaria, allorché questi tratti vengono riconosciuti come tali da una figura esterna. Una figura che deve però essere a sua volta riconosciuta dal primo soggetto, e il cui sguardo può così essere traslato all'interno della sua coscienza, o meglio della sua autocoscienza. Una volta interiorizzato lo sguardo esterno, questa modalità particolare di rapportarsi al soggetto diviene parte integrante del soggetto stesso, che pertanto definisce e amplia la propria struttura identitaria.

È paradigmatico di tale modello hegeliano del reciproco riconoscimento il caso dell'amore: soltanto quando un soggetto viene amato, esso può percepire a sua volta se stesso come un soggetto bisognoso di amore. Da quel momento, l'amore e il desiderio del soggetto stesso non devono pertanto essere intesi come un semplice desiderio di possesso dell'altro, ma piuttosto quale desiderio che l'altro desideri, ami il soggetto. Dunque, come desiderio di essere desiderato. Generalizzando in modo sistematico tale approccio, Honneth (1992) può trarre «l'assunto teorico in base al quale lo sviluppo dell'identità personale di un soggetto è per principio connesso al presupposto di determinate modalità di riconoscimento da parte di altri soggetti» (p. 49). In breve, la relazione interpersonale qui in gioco «dischiude reciprocamente ai soggetti comunicanti la possibilità di esperire nel loro partner nella comunicazione come quel tipo di persona che essi a loro volta riconoscono». Ove però, a differenza della semplice tesi per cui l'identità personale deve transitare necessariamente at- 
traverso il riconoscimento intersoggettivo, nell'approccio hegeliano emerge come il soggetto debba a sua volta riconoscere il proprio partner: nella relazione di riconoscimento è «insito un obbligo alla reciprocità: i soggetti che si incontrano sono come costretti senza violenza a riconoscere in un determinato modo anche la loro controparte sociale» (Honneth, 1992, pp. 49-50).

Posti tali fondamenti, Honneth prosegue la sua analisi sistematica della concezione del reciproco riconoscimento del giovane Hegel, articolata nei tre stadi dell'amore, del diritto e della solidarietà, esplicitando infine la necessità di liberarla dai suoi corposi presupposti metafisici. Si tratta qui di svincolarsi dalla stilizzazione idealistica hegeliana dei rapporti intersoggettivi di reciproco riconoscimento per reinterpretarli quali eventi empirici che avvengono nel contesto complessivo del mondo sociale. È soltanto attraverso una reinterpretazione in grado di naturalizzare l'originario modello hegeliano che questo stesso modello può contribuire a dar vita a una teoria normativa della società in grado di far presa sulla realtà. A tal fine Honneth riprende la psicologia sociale di George Herbert Mead, e in particolare la raccolta (postuma) di lezioni intitolata Mente, sé e società (Mead, 1934). Partendo a sua volta da un retroterra hegeliano, Mead si muove infatti in direzione di una traduzione dell'originario linguaggio metafisico in termini naturalistici e materialistici, e viene pertanto a rappresentare un "ponte" che da Hegel riconduce al dibattito sociofilosofico attuale.

Nella rilettura dei processi di natura eminentemente intersoggettiva inerenti alla costruzione identitaria delineati da Mead attraverso i concetti basilari di Me, Sé e Io, Honneth insiste ripetutamente sul modello comunicativo e dialogico che ne rappresenterebbe uno dei fondamentali presupposti concettuali. Egli sottolinea ad esempio la rilevanza dei gesti vocali, rispetto ai quali i soggetti in via di sviluppo reagiscono a se stessi come i loro interlocutori, autocollocandosi in una prospettiva eccentrica, dalla quale possono pervenire a una determinata immagine di loro stessi e quindi a conquistare un certo grado di consapevolezza di sé. Sempre in questa direzione, Honneth (1992) rimarca altresì che il concetto di Me è teso anche a mostrare che «l'individuo può diventare consapevole di sé solo oggettivandosi; infatti, il Sé che esso ha davanti quando reagisce a se stesso è sempre il partner nell'interazione percepito dalla prospettiva del suo interlocutore» (p. 93), per poi sottolineare come la stessa relazione tra Me e Io sia «paragonabile al rapporto tra i partner in un dialogo» (ibidem). In breve, qui Mead approda a una concezione intersoggettiva dell'autocoscienza umana, incentrata sulla tesi per cui «un soggetto può acquisire consapevolezza di sé solo nella misura in cui impara a percepire il proprio agire dalla prospettiva, rappresentata simbolicamente, di una seconda persona» (ibid.), tale da dare un fondamento naturalistico alla teoria hegeliana del riconoscimento. 


\section{Intersoggettività primaria e conquista dell'autonomia}

Attraverso la ripresa di Mead, Honneth ha dunque naturalizzato in termini di reciproco riconoscimento il processo intersoggettivo di formazione dell'autocoscienza e delle diverse istanze intrapsichiche costitutive del soggetto su cui riposa la stessa dinamica di formazione dell'identità personale. Ė però soltanto grazie alla teoria delle relazioni oggettuali che egli può ora tentare di render conto di tali processi anche rispetto alla dimensione affettiva, concentrandosi in particolare sulle prime fasi di vita del bambino. Sul versante degli affetti primari, Honneth innesta infatti all'interno della propria teoria del riconoscimento il portato delle analisi sviluppate da Winnicott, e la correlata strumentazione concettuale (vedi soprattutto Winnicott, 1971). Si tratta quindi del primo stadio della tripartizione hegeliana del riconoscimento: quello inerente all'amore, inteso dalla prospettiva della soddisfazione e reciproca conferma dei bisogni affettivi, che secondo la formulazione di Hegel deve essere compreso come un "esser se stessi in un estraneo". Una definizione che Honneth interpreta come volta a indicare il precario equilibrio tra autonomia e dipendenza proprio dei legami d'amore, e che egli ritraduce direttamente nei termini della teoria delle relazioni oggettuali: come equilibrio tra simbiosi e autonomia. Ne consegue che «la tradizione di ricerca della teoria della relazione oggettuale è particolarmente adatta a far intendere l'amore come un rapporto di interazione basato su una particolare modalità di reciproco riconoscimento» (Honneth, 1992, p. 118). Ove è chiara la postura metodologica di fondo adottata: la psicoanalisi winnicottiana è qui adoperata quale elemento concettuale atto a rinforzare e a sorreggere, dall'interno ma grazie a una strumentazione di diversa natura, la tenuta del framework di matrice hegeliana del reciproco riconoscimento rilanciato da Honneth. Un atteggiamento che, facendo leva sulla prospettiva interpersonale e intersoggettivista, si accompagna nel contempo a una critica piuttosto radicale di Freud, accusato di aver adottato una prospettiva "monologica": «Per Freud e i suoi allievi i partner nell'interazione con il bambino erano significativi solo nella misura in cui comparivano come oggetti di un investimento libidico derivante dal conflitto intrapsichico fra le istanze pulsionali inconsce e i meccanismi di controllo che vengono insediandosi nell'Io». Se poi è vero che alla madre veniva riconosciuta una funzione più significativa, resta tuttavia - conclude Honneth (1992) - che in tal modo «veniva fissata un'immagine dello sviluppo psichico del bambino nella quale le relazioni con le altre persone erano considerate come semplici occasioni del manifestarsi delle pulsioni libidiche» (pp. 118-119). Viceversa, nella teoria delle relazioni oggettuali si assiste alla valorizzazione del processo di socializzazione del bambino, inteso dalla prospettiva dello sviluppo delle relazioni affettive, che conduce gradualmente alla conquista dell'autonomia. 
La ripresa di questa impostazione non concerne però semplicemente il fatto che essa presupponga un generico allargamento in direzione intersoggettivistica del processo di formazione identitaria sul versante affettivo. Più precipuamente, Honneth ritiene che la griglia concettuale volta a interpretare le relazioni affettive primarie quali forme di bilanciamento tra simbiosi e autoaffermazione, fusione e autonomia, possa essere intesa nei termini specifici della teoria del reciproco riconoscimento. Egli qui muove dall'assunzione di base di Winnicott secondo cui non ha alcun senso studiare un neonato isolandolo dalle sue relazioni interpersonali, e in particolare astraendo dal rapporto con la madre. Si deve invece procedere, al contrario, dalla relazione tra madre e neonato, che inizialmente assume una forma simbiotica, cioè una fase di intersoggettività indifferenziata, o primaria, o di unità indifferenziata. È a partire da questa sorta di unità originaria che viene delineato il compito della conquista dell'autonomia da parte del bambino, e della sua riconquista da parte della madre. Compito che assume la forma di un processo di separazione e di distacco dalla persona amata, che deve nel contempo condurre a una nuova forma di accettazione e amore reciproco. Come nel framework hegeliano, spicca la centralità giocata dalla relazione intersoggettiva tra madre e bambino, e non dei due poli presi singolarmente. Ed è sempre tenendo al centro la relazione in gioco che viene schizzato quel processo di graduale e reciproca emancipazione della madre e del bambino che dalla "dipendenza assoluta" giunge alla "dipendenza relativa".

L'accento è poi posto sulle forme attraverso cui il bambino cerca di elaborare, anche attraverso l'utilizzo di oggetti transizionali (Winnicott, 1951), la disillusione a lui inferta dal graduale riconoscimento della indipendenza della madre, e quindi dello scacco della sua presunta onnipotenza. Sulla falsariga della lettura proposta da Jessica Benjamin (vedi soprattutto Benjamin, 1988), l'analisi winnicottiana degli atti aggressivi che il lattante rivolge alla madre viene qui reinterpretata come una forma peculiare della lotta per il riconoscimento descritta da Hegel: si staglia una relazione di lotta giocata tra distruzione e dedizione amorevole, dipendenza e indipendenza reciproche. Insistendo sul tema degli oggetti transizionali, e sulla correlazione tracciata da Winnicott tra la fiducia nella dedizione materna e la capacità del bambino piccolo di stare e giocare da solo, Honneth rimarca poi come anche questa forma fondamentale di autorelazione, la fiducia in se stessi, rimandi direttamente alla dinamica della lotta per il riconoscimento. Viene infine sottolineato che estendendo e generalizzando l'approccio di Winnicott nella direzione suddetta, tutte le relazioni amorose possono essere ricondotte all'esperienza della simbiosi originaria tra madre e figlio, e dunque quali modalità relazionali dotate di una certa "tensione intersoggettiva". Tensione che spinge verso differenti modalità di equilibrio o di squilibrio, anche patologico, tra il desiderio di fusione e la volontà di continuare a veder riconosciuti i confini del Sé, proprio e altrui, e dunque tra autonomia e simbiosi, indipendenza e dipendenza, ego-centrismo e 
de-centramento. Dinamiche che sul piano metodologico dovrebbero infine confermare, più o meno indirettamente, la cogenza di una riformulazione dei rapporti affettivi primari dischiusi dalla teoria delle relazioni oggettuali quali forme specifiche di lotte per il riconoscimento. Riformulazione collocata a sua volta in un quadro complessivo che mira a riattualizzare il modello teorico hegeliano di riferimento.

\section{La psicoanalisi intesa in termini di teoria del riconoscimento}

\section{Spazi comunicativi intrapsichici dialogici}

A partire dal saggio "Teoria delle relazioni oggettuali e identità postmoderna”, del 1999, Honneth avanza una proposta volta a tradurre sistematicamente una molteplicità di processi intrapsichici in termini di relazioni di comunicazione e di reciproco riconoscimento. L'organizzazione di una serie di eventi psicologici e le correlazioni tra le istanze interne che li determinano può e deve essere compresa ricollocando tali fenomeni entro un quadro dinamico il cui funzionamento di base risulta analogo a quello che, seguendo i dettati della teoria del riconoscimento, è desumibile dalla realtà sociale. Uno spazio sociale della ragione dialogica trasposto all'interno della psiche, si potrebbe forse dire. Tale proposta mira anche a far fronte alla sfida interpretativa lanciata ai modelli concettuali della psicoanalisi tradizionale dal portato che i cambiamenti delle nostre attuali società imprimono alla dimensione spirituale, e in particolare nei confronti dell'orientamento normativo verso un determinato modello di salute mentale; dunque rispetto alle questioni brucianti e convergenti dell’identità personale e dello sviluppo dell’'Io.

Honneth si contrappone alla tesi sempre più diffusa di una presunta obsolescenza della psicoanalisi, secondo cui essa non sarebbe appunto in grado di decodificare quelle profonde trasformazioni psichiche co-determinate dalla polverizzazione di tutta una serie di figure e ruoli tradizionali. A iniziare dalla spinta socioculturale determinata dalla rivoluzione sessuale, si assiste infatti a una continua ridefinizione e fluidificazione di una serie di correlazioni sociali, familiari e professionali che poggiavano su salde e relativamente rigide distinzioni convenzionali. Processi che hanno contribuito alla nascita di una "personalità postmoderna" che Honneth caratterizza essenzialmente nei termini invero soltanto vagamente accennati - di una pluralizzazione intrapsichica dei soggetti. Processo che a suo avviso non deve però essere ridotto e liquidato interpretandolo unilateralmente quale forma negativa di declino del Sé, o di perdita di identità, né tantomeno quale fenomeno che decreterebbe la definitiva obsolescenza del pensiero psicoanalitico nel suo insieme. Al contrario, il processo di de-tradizionalizzazione in gioco deve essere inteso quale ampliamento positivo della gamma, della ricchezza delle possibilità di sviluppo interno, e può essere colto e compreso grazie ad alcuni strumenti concettuali of- 
ferti dalle versioni più avanzate della psicoanalisi, ibridate alla teoria del riconoscimento. Qui viene infatti sostenuta esplicitamente la necessità di «delineare brevemente le premesse iniziali di una psicoanalisi intesa in termini di teoria del riconoscimento» (Honneth, 1999, p. 17).

Honneth procede alla ripresa e rilettura di quegli sviluppi della teoria delle relazioni oggettuali volti a interpretare lo sviluppo psichico nei termini di una graduale interiorizzazione di relazioni interpersonali, dunque secondo un modello comunicativo, ora in grado di render conto della formazione identitaria infantile. Di contro allo sviluppo endogeno proposto da Freud, in tale tradizione interazionista anche le forme di organizzazione pulsionale vengono messe in rapporto diretto con i processi di interiorizzazione nel quadro complessivo delle linee di sviluppo dell'Io. In altri termini, si tratta ancora di riprendere e coniugare i due modelli teorici desunti dalla psicologia sociale di Mead e dalla psicoanalisi di Winnicott alla luce della teoria del riconoscimento, modelli secondo i quali il meccanismo fondamentale della interiorizzazione viene a determinare sia il processo di socializzazione sia quello di individuazione. Su un versante, la riuscita interiorizzazione di una relazione interpersonale depotenzia l'impatto che le forze esterne esercitano sull'individuo, consolidandone la relativa autonomia. Sull'altro versante, il medesimo processo amplia le possibilità di articolazione e differenziazioni interne dei bisogni e fini individuali, favorendo l'acquisizione di una sempre maggiore autonomia psichica nel quadro di una graduale moltiplicazione delle relazioni interpersonali. È in tal senso che vengono rilette la costruzione del "me" in Mead, e quella dello stare "da solo" e della creatività in Winnicott (1971). Viceversa, la dimensione intrapsichica che resta immune da tali forme di interiorizzazione è rappresentata da quel serbatoio di energie pulsionali racchiuse negli spazi definitivi rispettivamente dell'Io (Mead) e dell'Es (Freud/Winnicott); regioni da cui muovono le richieste inconsce che spingono ininterrottamente il soggetto a conquistare gradi sempre più alti d'integrazione e autonomia, secondo un modello relazionale di stampo fondamentalmente dialogico.

Tracciate le analogie strutturali tra il modello di Mead e quello di Winnicott dal punto di vista delle strutture comunicative intrapsichiche, Honneth insiste sulla peculiarità e forza esplicativa dell'approccio psicoanalitico delle relazioni oggettuali, tale per cui esso può e deve esser considerato in grado di rispondere esaustivamente alla sfida dell'identità postmoderna. La tesi portante di Winnicott qui rilanciata è che il processo infantile di interiorizzazione delle relazioni esterne è preceduto da una periodo di unione indifferenziata tra neonato e realtà, nonché di simbiosi tra lui e la madre, sì che il bambino deve affrontare il difficile compito di separarsi affettivamente dalla madre e giungere sincronicamente a riconoscere l'indipendenza del mondo esterno. Viene qui schizzato anche il ruolo degli oggetti transizionali (Winnicott, 1951), e più in generale il processo di costruzione di una zona intermedia, dall'ambivalen- 
te status ontologico, che permette al bambino di risolvere tale compito, seppur dolorosamente, e che da qui in avanti lascia aperta la via alla possibilità di perdere o sospendere periodicamente tale separazione: di ritornare in forme diverse alla zona intermedia transizionale.

Per Honneth la psicoanalisi può inoltre contribuire in modo significativo alla costruzione di un modello ideale di personalità anche dal punto di vista di una aggiornata teoria pulsionale. È a questo riguardo che viene ripresa l'impostazione di Hans Loewald (1980) volta a offrire una lettura intersoggettiva della formazione del potenziale pulsionale o istintuale. Muovendo dalla tesi organicista di una originaria mancanza di forme e strutture dei materiali pulsionali, Loewald sostiene che è solo grazie alla relazione accudente della madre che il bambino inizia a dare una forma a tali materiali, che vengono così a essere legati a oggetti specifici, e ad assumere pertanto i connotati propri delle pulsioni. Riprendendo la tesi di Winnicott dello stato originario fusionale, Loewald può quindi presentare il processo di separazione e di individuazione come una forma di differenziazione della vita pulsionale in diverse istanze, che a loro volta rappresentano il frutto della interiorizzazione di particolari modelli di interazione. L'Io e il Super-Io vengono così a configurarsi quali forme cristallizzate di energia pulsionale, liberata originariamente dal processo di separazione e che poi via via fluisce dall'Es, che continua a restare una sfera arcaica e poco organizzata. Honneth interpreta quindi tale modello seppur in modo piuttosto approssimativo - come se esso implicasse il fatto che Io e Super-Io non vengono più intesi come "forze contro gli istinti", ma come loro forme di organizzazione.

Tracciati tali lineamenti, Honneth trae alcune conclusioni di carattere generale, insistendo anzitutto sul fatto che la sua reinterpretazione delle linee di ricerca di Mead, Winnicott e Loewald viene a disegnare un processo di sviluppo tale per cui, «con un minimo di speculazione, si potrebbe dire che tutto ciò che costituisce la nostra vita interna - desideri, stimolazioni della coscienza, valutazioni della realtà e ideali - consiste nell'insieme di numerose voci che rappresentano forme più o meno solidificate di energia istintuale e, quando l'interiorizzazione è riuscita, si mettono in relazione l'una con l'altra in un modo che somiglia a un dialogo» (Honneth, 1999, p. 25). Ed è precisamente questo il modello dialogico che Honneth cerca di rilanciare nel quadro delle relazioni oggettuali, ponendolo quale alternativa allo schema tradizionale che insiste sulla "forza dell'Io" e del "controllo razionale". Approccio che dovrebbe così contribuire a determinare uno spostamento dell'orientamento normativo psicoanalitico tradizionale, e quindi a delineare un nuovo ideale di personalità. Anziché muovere nella direzione di un rafforzamento delle funzioni di controllo dell'Io, viene valorizzato e perseguito un atteggiamento intrapsichico eminentemente dialogico volto a preservare e a valorizzare la ricchezza, la vitalità e la pluralità della vita interiore. 


\section{Lotte e pulsioni}

La proposta volta a rivedere la metapsicologia psicoanalitica di orientamento relazionale alla luce del framework intersoggettivista della teoria del riconoscimento lanciata con decisione da Honneth alla fine degli anni 1990 è stata attaccata con altrettanta fermezza dal noto psicoanalista statunitense, nonché studioso di filosofia e teoria critica, Joel Whitebook ${ }^{1}$. Nel lungo articolo "Riconoscimento reciproco e lavoro del negativo", del 2001, egli ha difatti avanzato una dura critica a tale proposta, inserendola all'interno di una discussione volta a individuare alcuni limiti e talune falle della svolta intersoggettiva avviata da Habermas e proseguita appunto da Honneth. Discussione talmente di ampio respiro che talvolta il filo del discorso sembra invero perdersi nella trama. Emerge tuttavia con chiarezza che uno degli obiettivi di fondo perseguiti da Whitebook è difendere e rilanciare l'originaria antropologia politica freudiana presentata in testi quali Il disagio della civiltà (Freud, 1929), declinata ancora una volta nel quadro tradizionale hobbesiano sintetizzabile nel motto classicissimo homo homini lupus. Una antropologia negativa, dunque, segnata da una forte connotazione di taglio biologistico, tale per cui qualsivoglia processo di socializzazione deve sempre e comunque fare i conti con una dimensione pulsionale restia a essere addomesticata; sostanzialmente, «l'egoismo, l'aggressività e il desiderio di possesso», forze che sarebbero «sempre esistite come parti della nostra costituzione antropologica e psicologica» (Whitebook, 2001, p. 607). Da qui la necessità del "lavoro del negativo". Se gli sforzi di Whitebook si muovono quindi in una direzione non particolarmente originale, peraltro attraverso una analisi che non è esente da drastiche semplificazioni sul versante filosofico, credo risultino invece più interessanti alcuni aspetti delle critiche rivolte a Honneth, e in particolare quelle svolte lungo le due seguenti linee argomentative, tra loro correlate.

La prima linea concerne l'utilizzo della nozione di lotta per il riconoscimento delineata da Hegel. Whitebook insiste sul fatto che concentrandosi sugli scritti giovanili del periodo di Jena, ed escludendo la Fenomenologia dello spirito (Hegel, 1807), nella quale è sviluppata anche la celebre immagine della lotta per la vita e per la morte tra il servo e il signore, Honneth sarebbe approdato a una «radicale attenuazione della natura conflittuale e quindi dell'intensità della lotta per il riconoscimento» (Whitebook, 2001, p. 614). Egli insiste sul fatto che il reciproco riconoscimento non nasce da una precedente esperienza di reciprocità, ma è invece l'esito di una lotta in senso stretto, tale da costringere i soggetti a rinunciare all'utilizzo dell'altro come oggetto per i propri desideri, cioè quale oggetto narcisistico, e dunque a rinunciare

\footnotetext{
${ }^{1}$ Un articolo di Joel Whitebook, dal titolo "Psicoanalisi, religione e progetto di autonomia", è stato pubblicato a pp. 439-462 del n. 4/2010 di Psicoterapia e Scienze Umane. [N.d.R.]
} 
alla propria onnipotenza. Questo tipo di riconoscimento non deve pertanto essere inteso come «l'esito di un dialogo civile», né è perseguibile attraverso «mezzi puramente cognitivi» (Whitebook, 2001, p. 619): si tratta di una lotta per l'identità (non per l'autoconservazione) carica affettivamente.

La seconda linea argomentativa percorsa da Whitebook che trovo particolarmente significativa concerne anch'essa la questione del modello dialogico, ma è collocata nel quadro della ripresa della teoria delle relazioni oggettuali. La critica è incentrata sull'assunzione per cui il benessere psichico possa essere inteso nei termini di una "capacità intrapsichica di dialogo", ed è decisamente feroce: «Anche Freud parlò di "comunicazione", ma già allora il termine era troppo impreciso per descrivere l'interazione (o il rapporto) tra le istanze. L'assimilazione, poi, della "comunicazione” al “dialogo" - con le sfumature di moderazione, non violenza e simmetria presenti in quest'ultimo - è assolutamente fuorviante». Fuorviante perché - spiega Whitebook riprendendo Green - l'“ideale neutrale di simmetria” del modello dialogico disconosce quella fondamentale "polemica" tra dimensione conscia e inconscia di cui la psicoanalisi dovrebbe invece render conto; polemica che presenta la forma di una «lotta per il potere» (Whitebook, 2001, p. 631).

$\mathrm{Al}$ di là di una certa unilateralità e vaghezza concettuale, nonché di una sorta di infelice commistione di piani analitici, a mio avviso Whitebook tocca uno dei nervi scoperti della proposta di Honneth: quello inerente al versante dei conflitti intrapsichici. In breve, lo schema di un reciproco riconoscimento sviluppato anche, seppur perlopiù tacitamente, alla luce del modello del dialogo consensuale ereditato dalla teoria dell'agire comunicativo di Habermas (1981), effettivamente contribuisce a generare una decisa depoliticizzazione delle dinamiche intrapsichiche, tale da depotenziare alla radice la capacità euristica degli strumenti di matrice psicoanalitica. Nel momento in cui lacerazioni, scissioni e fratture intrapsichiche potenzialmente patogene o direttamente patologiche vengono reinterpretate attraverso le griglie concettuali di forme di comunicazione e dialogo orientato all'intesa, non si può difatti che depotenziarne l'effettiva portata, distorcendo il senso stesso della psicoanalisi intesa quale strumento diagnostico. È come se reinterpretando l'obiettivo terapeutico tradizionale ideale di una invero sempre precaria e temporanea armonizzazione e riconciliazione delle istanze in conflitto nei termini del perseguimento di un dialogo consensuale tra "voci" differenti, Honneth sia più o meno involontariamente scivolato in un atteggiamento teorico che di fatto tende a proiettare gli obiettivi normativi sul piano analitico-esplicativo, determinando in tal modo una distorsione della percezione dei fenomeni in gioco. Il piano normativo tende a collassare su quello esplicativo.

Inoltre, allorché si consideri che questo modello dialogico delle dinamiche intrapsichiche è a sua volta il frutto di una sorta di proiezione del modello he- 
geliano delle lotte per il riconoscimento, si comprende come la medesima tendenza alla depoliticizzazione si riscontri anche sul versante della filosofia sociale. Anche in virtù della ricezione dell'eredità habermasiana del dialogo consensuale, nonché del teleologismo storico-filosofico tipicamente hegeliano, Honneth per taluni aspetti è stato indotto - dalla stesura di Lotta per il riconoscimento, del 1992, fino al più recente "Il diritto della libertà" (Honneth, 2011) - a depotenziare i caratteri radicalmente conflittuali delle grammatiche morali e delle correlate semantiche politiche di una molteplicità di lotte sociali storicamente date. Quasi come se ci si trovasse per assurdo di fronte a lotte sociali senza che mai debbano o possano darsi vinti e vincitori, dominati e dominatori, servi e signori, ma sempre parti perlomeno parzialmente reciprocamente e consensualmente riconoscentesi.

Nel prosieguo del loro confronto, Whitebook e Honneth si sono poi concentrati soprattutto su una serie di questioni inerenti alla dimensione presociale del Sé, con particolare riferimento alle pulsioni aggressive, alle tendenze all'onnipotenza e agli stati simbiotici negli infanti; senza pertanto affrontare le suddette questioni (Honneth, 2001b; Whitebook, 2003). Un approfondimento della questione inerente allo status della pulsione di morte, e più in generale dei rapporti tra teoria critica e forme della psicoanalisi, è invece rappresentato dall'articolo "Il lavoro del negativo" (Honneth, 2001a). Testo nel quale viene nel contempo rilanciato lo "stretto legame" proprio della tradizione francofortese tra il pensiero psicoanalitico e una teoria critica della società. Rimarcata l'obsolescenza socioculturale delle peculiari prospettive dalle quali la psicoanalisi freudiana era stata innestata nel corpo teorico di taglio marxista della prima teoria critica, Honneth spiega che, chiusa la parentesi habermasiana, sono in particolare due gli ordini di ragioni che spingono a risiglare e rinnovare tale alleanza teoretica. Il primo è di natura normativa. Una teoria critica della società che voglia disporre di un concetto di persona umana "realistico" deve contemplare in esso anche le forze e le energie irrazionali e inconsce; soltanto in questo modo può evitare di incagliarsi nelle secche dell'idealismo morale. Ma anche sul piano esplicativo, una teoria che voglia rendere conto in modo adeguato dei processi e delle relazioni sociali non può rinunciare a ricorrere anche al piano dei moti pulsionali inconsci che legano i soggetti e li spingono ad agire. Esigenze ottemperate grazie al ricorso alla teoria delle relazioni oggettuali: il miglior candidato per «un completamento psicologico della teoria critica, i cui concetti fondamentali consentono una traduzione relativamente immediata in categorie teoretico-sociali» (Honneth, 2001a, p. 255). Orientamento psicoanalitico considerato alla luce dei suoi sviluppi più recenti, tali da contemplare la presa in carico di fenomeni psicopatologici quali le sindromi borderline e i disturbi narcisistici, e che oltre al rapporto madre-figlio tengono conto di tutta una serie di altre figure fami- 
liari e sociali. Sulla falsariga dischiusa a suo tempo da Erich Fromm, tale indirizzo permette insomma una reinterpretazione complessiva dei processi di socializzazione di taglio identitario. Ed è da questa medesima prospettiva che Honneth attacca la matrice biologistica del dualismo pulsionale freudiano, optando per una reinterpretazione delle pulsioni aggressive in termini di processi di socializzazione e interiorizzazione.

\section{Il sostegno reciproco tra teoria del riconoscimento e psicoanalisi politica}

\section{Approcci diagnostici e normativi alle patologie sociali}

Con il saggio dedicato alla discussione critica della psicologia politica, o se vogliamo della psicoanalisi politica, di Franz Neumann (1889-1954), poi con quello sui processi regressivi inerenti alle dinamiche pulsionali e ai funzionamenti dei gruppi sociali, Honneth prosegue nel suo progetto di rilanciare l'originario progetto interdisciplinare della prima teoria critica francofortese volto a saldare psicoanalisi e teoria sociale, rivedendone e riaggiornandone in profondità sia il versante psicoanalitico sia quello dell'analisi sociale e politica (Honneth, 2002, 2003). Quanto al primo versante, la teoria critica di Theodor Adorno e Herbert Marcuse aveva nettamente prediletto Freud e "I'ortodossia freudiana", schierandosi frontalmente contro tutte quelle letture che, a iniziare da Fromm, avevano cercato di promuoverne delle interpretazioni eterodosse, nonché più vicine all'indirizzo della psicologia sociale statunitense. Honneth muove ora in direzione inversa: tenendosi a distanza dal freudismo, insiste sulla teoria delle relazioni oggettuali quale via che viene a convergere con una serie di elementi e modelli teoretici della psicologia sociale. Ed è pertanto tale tradizione psicoanalitica che egli cerca, anche da questa prospettiva sociopolitica, di reinnestare nel corpo di una critica sociale ancorata alla teoria del riconoscimento. Una teoria critica della società, quindi, che non rinuncia a sviluppare esplicitamente e sistematicamente la propria dimensione normativa in direzione di una visione positiva dello stato democratico di diritto atta nel contempo a diagnosticarne le patologie.

In uno degli ultimi lavori di Neumann (1954), lo scritto intitolato "Angoscia e politica”, Honneth trova una tematizzazione dell'angoscia nevrotica che gli permette di posizionare tale concetto nella zona di intersezione tra il piano psicosociale e quello politico normativamente orientato. Egli riprende infatti l'intuizione per cui soltanto dei cittadini liberi da angosce irrazionali, immotivate, e quindi "liberi dalla paura", godono di un grado di autonomia individuale tale da consentirgli di contribuire effettivamente al processo riflessivo di formazione della volontà democratica, e quindi, più in generale, al corretto funzionamento di uno stato democratico di diritto. Viceversa, allorché le an- 
gosce nevrotiche superano il limite suddetto, paralizzando o rendendo apatici i cittadini, esse debbono essere considerate come forze patogene che non soltanto ledono l'autonomia individuale e il suo portato sociopolitico, ma che possono contribuire a innescare dei processi regressivi di massa. È questo l'approccio di "psicologia politica" che Honneth intende adottare, seppur in modo critico rispetto alle specifiche analisi proposte da Neumann, di cui propone una revisione sistematica che si concentra su due assi principali: il riferimento alla sola metapsicologia freudiana e la limitazione dell'analisi al caso del nazionalsocialismo.

Sul versante psicoanalitico, Honneth critica la nozione freudiana tradizionale di angoscia nevrotica ripresa da Neumann anzitutto perché intesa in termini di rimozione e direttamente correlata alla tesi della eccedenza pulsionale. Di contro, egli adotta quella accezione di angoscia nevrotica intesa come formazione reattiva secondaria, che nel quadro teorico delle relazioni oggettuali è ancorata al pericolo di una perdita di sostegni intersoggettivi, e che trova il suo modello paradigmatico e originario nella separazione del neonato dalla madre (o dalla persona di riferimento primaria). Egli pertanto riconduce l'angoscia alle difficoltà affettive che il bambino ha nel riconoscere l'indipendenza dell'altro. Proseguendo lungo questa via - sostiene Honneth - si può pervenire a una concezione differenziata dei diversi modelli di elaborazione intrapsichica di quelle esperienze della perdita che minacciano le relazioni intersoggettive ben più ricco e consono alle premesse metodologiche della psicologia politica di Neumann rispetto al modello originario freudiano. Sul versante dei processi psicosociali di massa, Honneth sottolinea come Neumann proceda riprendendo la classica visione della identificazione proiettiva, distingua poi tra identificazioni razionali e affettive o libidiche, e differenzi tra le dinamiche inerenti ai piccoli gruppi cooperativi o invece alle grandi masse guidate da leader carismatici. Honneth procede quindi nella critica, rimarcando anzitutto che la prima distinzione, da inscrivere nel solco del razionalismo psicologico di Max Horkheimer, è fallace: non si danno identificazioni nelle dinamiche di gruppo che non siano nel contempo razionali ed emotive, così come non si danno gruppi uniti dalla mera somma di interessi razionali. Allo stesso modo, anche la tematizzazione delle forme regressive viene a essere sbilanciata e unilaterale: qui Honneth sottolinea come una serie di forme di regressione individuale debbono essere intese quali aspetti positivi, salutari, di dissoluzione transitoria dei limiti dell'Io, come emerge ad esempio nella fusione con la persona amata o nell'immersione nel gioco.

Viceversa, Honneth valorizza la tesi di Neumann per cui tra i fattori sociali che possono contribuire a innescare dei processi regressivi di massa che conducono all'identificazione con un leader vi sia una angoscia sociale determinata dalla perdita di stima di sé. Tale offesa dell'autostima viene però sincro- 
nicamente ricondotta al quadro della teoria delle relazioni oggettuali, nel senso che le angosce sociali vengono ora a delinearsi come «forme mature e riattivate per via di esperienze di deprivazione di quelle angosce infantili traumatizzanti che si sono formate in quanto reazioni alla perdita della presenza costante, e donatrice di sicurezza, della persona di riferimento primario» (Honneth, 2002, p. 196). Infine, Honneth mostra come l'analisi di Neumann sia di fatto limitata al caso del nazionalsocialismo, nonostante il suo approccio miri a tematizzare le angosce di massa come forme di patologie sociali che ledono l'autonomia individuale, e dunque dischiuda la via a una diagnosi «normativamente fondata e coerente con i presupposti di una sfera pubblica democratica» (Honneth, 2002, p. 198).

La questione delle forme regressive e più in generale della forza esercitata da pulsioni e angosce nel quadro delle dinamiche dei gruppi sociali è ancora al centro dell'attenzione nel saggio "L’Io nel Noi” (Honneth, 2003). Qui Honneth propone un approccio alla tematizzazione del concetto di gruppo alternativa rispetto ai principali indirizzi teorici tradizionali novecenteschi, ora incentrato sul concetto di riconoscimento. Si tratta di trovare una terza via che eviti l'unilateralità delle critiche rivolte alle regressioni individuali nella massa, ancor oggi prevalente nell'area psicoanalitica, ma che eviti anche le secche dell'altrettanto unilaterale valorizzazione del portato civilizzatore dei processi di socializzazione, sia dei piccoli sia dei grandi gruppi, prevalente nella sociologia e nella teoria politica. Più da vicino, Honneth insiste sulla tesi per cui la dipendenza individuale dalle esperienze di riconoscimento sociale rende sincronicamente conto del fatto che i singoli aspirano a determinate forme di appartenenza nel quadro di gruppi specifici. Mentre le forme di riconoscimento via via conquistate nel corso dello sviluppo individuale risultano corrispondere a peculiari forme di raggruppamenti sociali. Tracciati quindi i tre gradi attraverso cui il bambino, per via di processi di socializzazione che conducono a diverse forme di interiorizzazione, giunge idealmente a sviluppare in sequenza fiducia in sé, rispetto di sé e autostima, e sincronicamente a inserirsi entro i gruppi sociali di riferimento, dalla famiglia ai suoi pari. Per correggere il tenore eccessivamente astratto e idealizzato di queste premesse, Honneth procede ora a una ritematizzazione delle tendenze regressive inconsce.

Ripreso il modello simbiotico tracciato da Winnicott, Honneth insiste sul fatto che la tendenza regressiva a voler superare i limiti dell'Io attraverso dei ritorni temporanei a stadi fusionali più o meno intensi - nei quali sfuma quindi il confine tra realtà interna ed esterna, come quelli rinvenibili secondo lo stesso Winnicott in relazione alle sfere dell'arte e della religione - può essere traslata, e quindi ampliata, anche rispetto a taluni fenomeni inerenti all'appartenenza ai gruppi sociali. Da qui l'accezione positiva conferita ai processi di regressione in gioco: «Queste fasi regressive, che solitamente si accompagna- 
no a tali esperienze di fusione, non sono indicatori di forme patologiche di riprimitivizzazione, ma piuttosto espressione di un recupero della vitalità psichica» (Honneth, 2003, p. 275). Nel contempo Honneth mette però in guardia da quelle dinamiche inerenti a proiezioni e fantasie inconsce che possono non soltanto influenzare il comportamento nel gruppo, ma condurre a una patologizzazione e distruzione dei processi comunicativi. Al riguardo si insiste però sul ruolo determinante giocato dai particolari tipi di individualità dei singoli componenti dei gruppi, e dei loro eventuali disturbi mentali, rovesciando così l'ordine tradizionale dei fattori.

\section{Libertà}

Con il saggio "Democrazia e libertà interiore" (Honneth, 2006a) viene ripreso il filo del discorso sull'angoscia quale fattore che, limitando potenzialmente l'autonomia individuale, può rappresentare una fonte patologica anche dal punto di vista politico. Honneth ritorna pertanto sul legame tra la debolezza dell'Io e il corretto funzionamento di uno stato democratico. L'analisi però verte ora sull'opera dello psicoanalista tedesco Alexander Mitscherlich (19081982) che, collocata l'angoscia nel più ampio quadro dell'antropologia filosofica novecentesca, la intende come una delle diverse forme patologiche che limitano dall'interno la libertà individuale. Di contro a tutte quelle forme che inducono a rimuovere, somatizzare e più in generale a sfuggire ai propri conflitti interiori adottando meccanismi di proiezione o di spostamento, tali da generare derive psicopatologiche politicamente pericolose, Honneth sottolinea come Mitscherlich nel corso del tempo abbia proposto a più riprese un modello di gestione delle forze ed energie psichiche potenzialmente patogene saldamente improntato alla tolleranza interiore. Più precisamente, si tratta «di reagire alle energie pulsionali che si presentano come pericolose non erigendo difese bensì con modalità magari all'inizio nate per gioco ma che poi gradualmente siano comprese come forme di "riconoscimento" (Honneth, 2006a, p. 207); Honneth riprende quindi direttamente il lessico adottato da Mitscherlich: «Il nostro autore chiama "tolleranza" e "libertà interiore" questa speciale capacità di accettazione di ciò che in me stesso, nelle forme di desideri, bisogni, struggimenti, risulta però estraneo a me stesso» (ibidem, p. 207). Honneth insiste quindi sul modello della comunicazione e della articolazione linguistica di tali elementi estranei, per poi stringere sul piano politico, riproiettando ora verso l'esterno il modello di tolleranza, e di ironia, prima importato all'interno, e saldando così l'isomorfismo: «Fino a quando saranno esigue le occasioni date per l'allargamento di simili forme di autorelazionarsi, fino a quando inoltre i soggetti rimarranno avvinghiati ai meccanismi dell'angoscia difensiva, persisteranno preponderanti la formazione del pregiudizio, la proiezione d'odio e l'esclusione sociale, tutte incompatibili con il compito della formazione discorsiva della volontà. Pertanto, il progetto di 
democratizzazione si riallaccia al presupposto di una condizione di libertà interiore, per la cui caratterizzazione il migliore modello è stato sinora offerto dalla psicoanalisi» (Honneth, 2006a, p. 209).

È sempre facendo leva sulla libertà, su un dispiegamento disinibito e scevro da angosce nevrotiche della libera volontà, che nel saggio " $L$ 'acquisizione della libertà" (Honneth, 2006b) viene ripreso e parzialmente rivalutato il progetto emancipatorio varato da Freud, e la visione antropologica che gli fa da sfondo. Il punto d'attacco è qui rappresentato dalla tesi per cui la psicoanalisi, anziché inseguire affannosamente gli sviluppi delle neuroscienze e aspettare ansiosamente conferme dalle sue ininterrotte scoperte, corre un pericolo mortale: «Perdere quell'ultimo elemento della teoria freudiana che la rende ancora fondamentalmente attuale e valida, al di là dei suoi aspetti divenuti discutibili: l'intuizione secondo cui l'uomo è anzitutto un essere scisso, interiormente tormentato, il quale però, grazie all'interesse ivi concesso di allargare la sua libertà interiore, possiede anche l'abilità di ridurre e a volte superare questa condizione di lacerazione per mezzo della sua attività riflessiva» (Honneth, 2006b, p. 168, corsivo nell'originale). Si tratta dunque di una visione antropologica complessiva, tale da aver cambiato - scrive Honneth - l'immagine tradizionale dell'essere umano, e che pone al centro dell'attenzione la questione cruciale dei fondamenti della relazione che egli instaura con se stesso. Una relazione che, attraverso la memoria, offre la possibilità di riconquistare pienamente, in modo riflessivo, la propria libera volontà. Honneth passa dunque a schizzare alcuni dei passaggi che condussero Freud a individuare tutta una serie di meccanismi patogeni presenti anche nei soggetti "sani” (sogni, atti mancati, lutto), presentando la sua opera quale tentativo volto fondamentalmente a revisionare la nostra idea di soggettività, piuttosto che a curare i nostri disturbi, per poi avvicinarsi alla tematizzazione dei processi di rimozione nel quadro delle dinamiche di sviluppo del bambino. E a questo punto che torna la questione dell'angoscia.

Premesso che Freud non sia inizialmente riuscito a render conto in modo adeguato della necessità di ricorrere alla rimozione per soggetti che hanno percorso dei processi di socializzazione del tutto normali, egli sarebbe infine approdato alla concezione dell'angoscia esposta in Inibizione, sintomo e angoscia (Freud, 1925). Qui l'angoscia infantile non è più intesa come se fosse causata dalla rimozione di particolari desideri ma, al contrario, è l'angoscia provata di fronte a particolari desideri che è ritenuta innescare il meccanismo della rimozione. Impostazione che conduce a individuare le origini di questa stessa angoscia infantile nel quadro di impronta "intersoggettiva" delle relazioni tra il neonato e la madre, verso la quale il primo si trova in una condizione di dipendenza assoluta. Da qui una fissazione "quasi biologica" nei confronti della madre e l'innesco di un segnale angosciante di pericolo di fronte alla possibilità di essere abbandonato. Schema relazionale che da qui in avanti 
verrà a costituire la modalità fondamentale emotiva reattiva alla paura di abbandono da parte dell'oggetto amato. Honneth sottolinea inoltre come il richiamo di Freud (1925) alla tesi per cui «il bisogno di essere amati» sia un «bisogno che non abbandonerà l'uomo mai più» (p. 301), abbia dischiuso la via poi percorsa da Winnicott (Honneth, 2006b, pp. 177-178). Delineato il passaggio per cui il bambino può reagire con angoscia, e quindi rimuovere quei desideri interiori che possono mettere in pericolo la relazione con la persona amata, Honneth prosegue nella sua reinterpretazione - che diviene invero piuttosto ardita - del compito freudiano di far fronte alle limitazioni dell'Io nei termini dall'accento spiccatamente etici e morali di un rafforzamento della "libera volontà". La natura emancipatoria del progetto freudiano verrebbe altresì a esplicarsi nel processo riflessivo attraverso cui, transitando per la memoria, l'individuo può costruire una relazione con se stesso tale da dischiudergli la possibilità di un recupero effettivo, non più soltanto ideale e normativo, delle sue capacità e facoltà. Honneth insiste infine sull'interpretazione dell'ermeneutica freudiana del Sé nei termini di un «dialogo interiore» nel quale il «processo di comunicazione» tra le diverse istanze intrapsichiche sarebbe tale da sottoporre a un «test dialogico» desideri e convinzioni da integrare a un certo livello (Honneth, 2006b, p. 183).

Honneth è poi ritornato a più riprese sul lascito freudiano e sulla sua rivalutazione. Certo ha continuato a ribadire la sua preferenza per Winnicott. Come ad esempio nel breve articolo "Esautorazioni della realtà" (Honneth, 2008), nel quale ha insistito sulla sua reinterpretazione sostanzialmente positiva di una serie di forme regressive rispetto alle quali Freud si era mostrato fondamentalmente ostile, a iniziare dal "sentimento oceanico", dalla fusione tra amanti e più in generala da quelle esperienze della prima infanzia nelle quali sfumano cioè i confini tra mondo interno e realtà esterna. Alla luce della teoria degli oggetti transizionali, le ricadute in stati fusionali, o meglio il passaggio attraverso la zona transizionale del reale, possono aiutare l'adulto a difendersi dalla pressione della realtà. Posto che, come sosteneva Winnicott, il compito di accettare la realtà esterna, e di porla in relazione con quella interna, è interminabile. E tuttavia, nonostante le critiche e le riserve, vi è un chiaro avvicinamento a Freud, come ha del resto dichiarato apertamente lo stesso Honneth. Ricapitolando alcune delle tappe del suo poliforme confronto e dei differenti usi della psicoanalisi realizzati nel corso del tempo, egli ha difatti rimarcato l'apprezzamento per un testo quale Inibizione, sintomo e angoscia (Freud, 1925), nel quale emerge una antropologia relativamente più attenta all'interazione interpersonale, nonché alla sfera negativa dell'angoscia (Honneth, 2009). Versante negativo che ha del resto ottenuto sempre maggior considerazione, sebbene la tematizzazione delle pulsioni aggressive continui a esser affrontata da una prospettiva fermamente socio-culturale e non meramente biologistica. 
Riassunto. Viene offerta una panoramica sui differenti usi e le diverse forme di psicoanalisi utilizzate da Axel Honneth in relazione alla sua "teoria del riconoscimento" nel corso del tempo. Dopo una discussione dell'uso della teoria delle relazioni oggettuali (soprattutto nella concezione di D.W. Winnicott) in Lotta per il riconoscimento, del 1992, si prende in esame la revisione della psicoanalisi operata in termini di teoria del riconoscimento. Infine viene discussa la proposta di una nuova alleanza tra una "teoria critica" rinnovata e la psicoanalisi, che contempla anche il versante della psicologia politica. [PAROLE CHIAVE: riconoscimento, psicoanalisi, intersoggettività, teoria delle relazioni oggettuali, Axel Honneth]

Abstract. Patterns of psychoanalysis and theory of recognition. Axel Honneth's intersubjective psyche. An overview of the several scopes and patterns used over time by Axel Honneth in his "theory of recognition" is presented. After a discussion of the use of object relations theory (especially with reference to D.W. Winnicott's contributions) in Honneth's 1992 book Struggle for Recognition, the theoretical revision of psychoanalysis in light of his theory of recognition is examined. Finally, Honneth's suggestion of a new alliance between a renewed "critical theory" and psychoanalysis, which concerns also the dimension of political psychology, is discussed. [KEY WORDS: recognition, psychoanalysis, intersubjectivity, object relations theory, Axel Honneth]

\section{Bibliografia}

Benjamin J. (1988). The Bonds of Love: Psychoanalysis, Feminism, and the Problem of Domination. New York: Pantheon (trad. it.: Legami d'amore: i rapporti di potere nelle relazioni amorose. Torino: Rosenberg \& Sellier, 1991; Milano: Raffaello Cortina, 2015).

Freud S. (1925 [1926]). Inibizione, sintomo e angoscia. Opere, 10: 233-317. Torino: Boringhieri, 1978.

Freud S. (1929 [1930]). Il disagio della civiltà. Opere, 10: 555-630. Torino: Boringhieri, 1978.

Habermas J. (1981). Theorie des kommunikativen Handelns. Frankfurt a.M.: Suhrkamp, 2 Bde. (trad. it.: Teoria dell'agire comunicativo. Bologna: Il Mulino, 1986, 2 volumi).

Hegel G.W.F. (1807). System der Wissenschaft. Erster Theil, Die Phänomenologie des Geistes. Bamberg Würzburg: Goebhardt (trad. it.: Fenomenologia dello spirito. Firenze: La Nuova Italia, 1996).

Honneth A. (1992). Kampf um Anerkennung. Zur moralischen Grammatik sozialer Konflikte. Frankfurt a.M.: Suhrkamp (trad. it.: Lotta per il riconoscimento. La grammatica morale dei conflitti sociali. Milano: Il Saggiatore, 2002).

Honneth A. (1999). Postmodern Identity and Object-Relations Theory: On the Supposed Obsolescence of Psychoanalysis. Philosophical Explorations, 2/3: 225-242 (trad. it.: Teoria delle relazioni oggettuali e identità postmoderna. Sulla presunta obsolescenza della psicoanalisi. Psiche. Rivista di cultura psicoanalitica, 2002, X, 1 [“Nuove identità”]: 3-27).

Honneth A. (2001a). Das Werk der Negativität. Eine psychoanalytische Revision der Anerkennungstheorie. In: Bohleber W. \& Drews S., editors, Die Gegenwart der Psychoanalyse Die Psychoanalyse der Gegenwart. Stuttgart: Klett-Cotta, 2001, pp. 238-245.

Honneth A. (2001b). Facetten des vorsozialen Selbst. Eine Erwiderung auf Joel Whitebook. Psyche. Zeitschrift für Psychoanalyse und ihre Anwendungen, 55, 8: 790-801.

Honneth A. (2002). "Angst und Politik” - Stärken und Schwächen der Pathologiediagnose von Franz Neumann. In: Iser M. \& Strecker D., editors, Kritische Theorie der Politik. Franz Neumann - eine Bilanz. Baden-Baden: Nomos, 2002, pp. 200-220 (trad. it.: “Angoscia e politica” - Franz Neumann: forza e debolezza della sua diagnosi delle patologie. In: Honneth, 2007, pp. 189-199). 
Honneth A. (2003). Das Ich im Wir. Anerkennung als Triebkraft von Gruppen. Jahrbuch für Gruppenanalyse. Heidelberg: Mattes, 2003, Bd. 9 (Ringen um Anerkennung in und zwischen Gruppen), pp. 5-22.

Honneth A. (2006a). Demokratie und innere Freiheit. Alexander Mitscherlichs Beitrag zur kritischen Gesellschaftstheorie. In: Drews S., editor, Freud in der Gegenwart. Alexander Mitscherlichs Gesellschaftskritik. Frankfurt a.M.: Brandes \& Apsel, 2006, pp. 94-102 (trad. it.: Democrazia e libertà interiore. Il contributo di Alexander Mitscherlich alla teoria critica della società. In: Honneth, 2007, pp. 201-209).

Honneth A. (2006b). Aneignung von Freiheit. Freuds Konzeption der individuellen Selbstbeziehung. WestEnd. Neue Zeitschrift für Sozialforschung, 2: 32-47 (trad. it.: L'acquisizione della libertà. La concezione freudiana dell'autorelazionarsi individuale. In: Honneth, 2007, pp. 167-188).

Honneth A. (2008). Entmächtigungen der Realität. Säkulare Formen des Trostes. In: Peters T.R. \& Urban C., editors, Über den Trost. Ostfildern: Grünewald, 2008, pp. 9-25.

Honneth A. (2009). Interview with Axel Honneth, by Inara Luisa Marin. European Journal of Psychoanalysis: www.journal-psychoanalysis.eu/interview-with-axel-honneth.

Honneth A. (2011). Das Recht der Freiheit. Grundriss einer demokratischen Sittlichkeit. Berlin: Suhrkamp.

Honneth A. (2007). Pathologien der Vernunft. Geschichte und Gegenwart der Kritischen Theorie, Frankfurt a.M.: Suhrkamp (trad. it. Patologie della ragione. Storia e attualità della teoria critica. Lecce: Pensa MultiMedia).

Loewald H.W. (1980). Papers on Psychoanalysis. New Haven, CT: Yale Univ. Press (trad. it. parziale: Riflessioni psicoanalitiche. Milano: Dunod Masson, 1999; trad. it. dei capitoli 4 e 14 [13 nella trad. it. Dunod Masson] anche in: Psicoterapia e Scienze Umane, rispettivamente n. 1/1993 [cap. 4] e n. 4/1993 [cap. 14, I parte] e n. 1/1994 [cap. 14, II parte]).

Mead G.H. (1934). Mind, Self and Society. Chicago, IL: University of Chicago Press (trad. it.: Mente, sé e società. Firenze: Giunti Barbera, 1966, 2010).

Neumann F. (1954). Angst und Politik: Vortrag gehalten an der Freien Universität Berlin aus Anlass der Verleihung der Wurde eines Ehrendoktors der philosophischen Fakultät. Recht und Staat, 178/179 (Tübingen: Mohr) (trad. it.: Angoscia e politica. In: Lo stato democratico e lo stato autoritario. Bologna: Il Mulino, 1973, pp. 113-147).

Whitebook J. (2001). Wechselseitige Anerkennung und die Arbeit des Negativen. Psyche. Zeitschrift für Psychoanalyse und ihre Anwendungen, 55, 8: 755-789 (trad. it.: Riconoscimento reciproco e lavoro del negativo. Rivista di Psicoanalisi, 2002, XLVIII, 3: 603-635).

Whitebook J. (2003). Die Grenzen des 'intersubjective turn’. Eine Erwiderung auf Axel Honneth. Psyche. Zeitschrift für Psychoanalyse und ihre Anwendungen, 57, 3: 250-261.

Winnicott D.W. (1951). Transitional objects and transitional phenomena: A study of the first not-me possession. International Journal of Psychoanalysis, 1953, 34, 2: 89-97. Anche in: Collected Papers. Through Pediatrics to Psychoanalysis. New York: Basic Books, 1958, pp. 229-242 (trad. it.: Oggetti transizionali e fenomeni transizionali. In: Dalla pediatria alla psicoanalisi. Scritti scelti. Firenze: Martinelli, 1975, cap. XVIII, pp. 275-290. Anche in: Winnicott, 1971, cap. 1, pp. 23-60).

Winnicott D.W. (1971). Playing and Reality. London: Tavistock (trad. it.: Gioco e realtà. Roma: Armando, 1974). 